\title{
OPTIMIZED HIGH-ORDER SPLITTING METHODS FOR SOME CLASSES OF PARABOLIC EQUATIONS
}

\author{
S. BLANES, F. CASAS, P. CHARTIER, AND A. MURUA
}

\begin{abstract}
We are concerned with the numerical solution obtained by splitting methods of certain parabolic partial differential equations. Splitting schemes of order higher than two with real coefficients necessarily involve negative coefficients. It has been demonstrated that this second-order barrier can be overcome by using splitting methods with complex-valued coefficients (with positive real parts). In this way, methods of orders 3 to 14 by using the Suzuki-Yoshida triple (and quadruple) jump composition procedure have been explicitly built. Here we reconsider this technique and show that it is inherently bounded to order 14 and clearly sub-optimal with respect to error constants. As an alternative, we solve directly the algebraic equations arising from the order conditions and construct methods of orders 6 and 8 that are the most accurate ones available at present time, even when low accuracies are desired. We also show that, in the general case, 14 is not an order barrier for splitting methods with complex coefficients with positive real part by building explicitly a method of order 16 as a composition of methods of order 8 .
\end{abstract}

\section{INTRODUCTION}

In this paper, we consider linear evolution equations of the form

$$
\frac{d u}{d t}(t)=A u(t)+B u(t), \quad u(0)=u_{0},
$$

where the (possibly unbounded) operators $A, B$ and $A+B$ generate $C^{0}$ semi-groups for positive $t$ over a finite or infinite Banach space $X$. Equations of this form are encountered in the context of parabolic partial differential equations, a prominent example being the inhomogeneous heat equation

$$
\frac{\partial u}{\partial t}(x, t)=\Delta u(x, t)+V(x) u(x, t),
$$

where $t \geq 0, x \in \mathbb{R}^{d}$ or $x \in \mathbb{T}^{d}$ and $\Delta$ denotes the Laplacian in $x$.

Received by the editor February 1, 2011 and, in revised form, October 13, 2011 and December 2,2011 .

2010 Mathematics Subject Classification. Primary 65L05, 65P10, 37M15.

Key words and phrases. Composition methods, splitting methods, complex coefficients, parabolic evolution equations.

The work of the first, second and fourth authors was partially supported by Ministerio de Ciencia e Innovación (Spain) under the coordinated project MTM2010-18246-C03 (co-financed by FEDER Funds of the European Union). Financial support from the "Acción Integrada entre España y Francia" HF2008-0105 was also acknowledged.

The fourth author was additionally funded by project EHU08/43 (Universidad del País Vasco/Euskal Herriko Unibertsitatea). 
A method of choice for solving numerically (1.1) consists in advancing the solution alternatively along the exact (or numerical) solutions of the two problems

$$
\frac{d u}{d t}(t)=A u(t) \quad \text { and } \quad \frac{d u}{d t}(t)=B u(t) .
$$

Upon using an appropriate sequence of steps, high-order approximations can be obtained - for instance, with exact flows - as

$$
\Psi(h)=\mathrm{e}^{h b_{0} B} \mathrm{e}^{h a_{1} A} \mathrm{e}^{h b_{1} B} \cdots \mathrm{e}^{h a_{s} A} \mathrm{e}^{h b_{s} B} .
$$

The simplest example within this class is the Lie-Trotter splitting

$$
\mathrm{e}^{h A} \mathrm{e}^{h B} \quad \text { or } \quad \mathrm{e}^{h B} \mathrm{e}^{h A},
$$

which is a first order approximation to the solution of (1.1), while the symmetrized version

$$
S(h)=\mathrm{e}^{h / 2 A} \mathrm{e}^{h B} \mathrm{e}^{h / 2 A} \quad \text { or } \quad S(h)=\mathrm{e}^{h / 2 B} \mathrm{e}^{h A} \mathrm{e}^{h / 2 B}
$$

is referred to as Strang splitting and is an approximation of order 2.

The application of splitting methods to evolutionary partial differential equations of parabolic or mixed hyperbolic-parabolic type constitutes a very active field of research. For this class of problems it makes sense to split the spatial differential operator, each part corresponding to a different physical contribution (e.g., reaction and diffusion). Although the formal analysis of splitting methods in this setting can be carried out by power series expansions (as in the case of ordinary differential equations), several fundamental difficulties arise, however, when establishing convergence and rigorous error bounds for unbounded operators HKLR10. Partial results exist for hyperbolic problems [TT95, Tang98, HKLR10, parabolic problems DS02, HV03, and for the Schrödinger equation [JL00, Lub08], even for high order splitting methods [Tal08.

In $\mathrm{HO} 09 \mathrm{a}$, it has been established that, under the two conditions stated below, a splitting method of the form (1.2) is of order $p$ for problem (1.1) if and only if it is of order $p$ for ordinary differential equations in finite dimension. In other words, if and only if the difference $\Psi(h)-\mathrm{e}^{h(A+B)}$ admits a formal expansion of the form

$$
\Psi(h)-\mathrm{e}^{h(A+B)}=h^{p+1} E_{p+1}+h^{p+2} E_{p+2}+\cdots .
$$

The two referred conditions write (see HO09a for a complete exposition):

(1) Semi-group property: $A, B$ and $A+B$ generate $C^{0}$ semi-groups on $X$ and, for all positive $t$,

$$
\left\|\mathrm{e}^{t A}\right\| \leq \mathrm{e}^{\omega_{A} t}, \quad\left\|\mathrm{e}^{t B}\right\| \leq \mathrm{e}^{\omega_{B} t} \quad \text { and } \quad\left\|\mathrm{e}^{t(A+B)}\right\| \leq \mathrm{e}^{\omega t}
$$

for some positive constants $\omega_{A}, \omega_{B}$ and $\omega$.

(2) Smoothness property: For any pair of multi-indices $\left(i_{1}, \ldots, i_{m}\right)$ and $\left(j_{1}, \ldots, j_{m}\right)$ with $i_{1}+\cdots+i_{m}+j_{1}+\cdots+j_{m}=p+1$, and for all $t \in[0, T]$,

$$
\left\|A^{i_{1}} B^{j_{1}} \ldots A^{i_{m}} B^{j_{m}} \mathrm{e}^{t(A+B)} u_{0}\right\| \leq C
$$

for a positive constant $C$.

However, designing high-order splitting methods for (1.1) is not as straightforward as it might seem at first glance. As a matter of fact, the operators $A$ and $B$ are only assumed to generate $C^{0}$ semi-groups (and not groups). This means in particular that the flows $\mathrm{e}^{t A}$ and/or $\mathrm{e}^{t B}$ may not be defined for negative times (this is indeed the case, for instance, for the Laplacian operator) and this prevents the use of 
methods which embed negative coefficients. Given that splitting methods with real coefficients must have some of their coefficients $a_{i}$ and $b_{i}$ negative 1 to achieve order 3 or more, this seems to indicate, as it has been believed for a long time within the numerical analysis community, that it is only possible to apply exponential splitting methods of at most order $p=2$. In order to circumvent this order-barrier, the papers $\mathrm{HO} 09 \mathrm{~b}$ and CCDV09 simultaneously introduced complex-valued coefficients 2 with positive real parts. It can indeed be checked in many situations that the propagators $\mathrm{e}^{z A}$ and $\mathrm{e}^{z B}$ are still well defined in a reasonable distribution sense for $z \in \mathbb{C}$, provided that $\Re(z) \geq 0$. Using this extension from the real line to the complex plane, the authors of [HO09b] and [CCDV09] built up methods of orders 3 to 14 by considering a technique known as triple-jump composition 3 that was made popular by a series of authors: Creutz \& Gocksch [CG89, Forest For89, Suzuki Suz90, and Yoshida Yos90.

In this work, we continue the search for new methods with complex coefficients with positive real parts. Eventually, our objective is to show that, compared to the methods built in [HO09b] and CCDV09] by applying the triple-jump (or quadruplejump) procedure, it is possible to construct more efficient methods and also of higher order by solving directly the polynomial equations arising from the order conditions. In particular, we construct methods of order 6 and 8 with minimal local error constants among the methods with minimal number of stages. We also construct a method of order 16, obtained as a composition based on an appropriate eighth-order splitting method.

Here we are particularly interested in obtaining new splitting methods for reaction-diffusion equations. These constitute mathematical models that describe how the population of one or several species distributed in space evolves under the action of two concurrent phenomena: reaction between species in which predators eat prey and diffusion, which makes the species spread out in space 4 . From a mathematical point of view, they belong to the class of semi-linear parabolic partial differential equations and can be represented in the general form

$$
\frac{\partial u}{\partial t}=D \Delta u+F(u)
$$

where each component of the vector $u(x, t) \in \mathbb{R}^{d}$ represents the population of one species, $D$ is the real diagonal matrix of diffusion coefficients and $F$ accounts for all local interactions between species 5 Strictly speaking, the theoretical framework introduced in [HO09b] does not cover this situation if $F$ is nonlinear, so that (apart

\footnotetext{
${ }^{1}$ The existence of at least one negative coefficient was shown in [She89, Suz91], and the existence of a negative coefficient for both operators was proved in GK96]. An elementary proof can be found in $\mathrm{BC} 05$.

${ }^{2}$ Methods with complex-valued coefficients have also been used in a similar context Ros63] or in celestial mechanics Cha03.

${ }^{3}$ And its generalization to quadruple-jump.

${ }^{4}$ Apart from biology and ecology, systems of this sort also appear in chemistry (hence the term reaction), geology and physics.

${ }^{5}$ The choice $F(u)=u(1-u)$ yields Fisher's equation and is used to describe the spreading of biological populations; the choice $F(u)=u\left(1-u^{2}\right)$ describes Rayleigh-Benard convection; the choice $F(u)=u(1-u)(u-\alpha)$ with $0<\alpha<1$ arises in combustion theory and is referred to as the Zeldovich equation.
} 
from Section 4 where we successfully integrate numerically an example with nonlinear $F$ ) we will think of $F$ as being linear. The important feature of $A=D \Delta$ here is that it has a real spectrum; hence, any splitting method involving complex steps with positive real part is suitable for that class of problems. In principle, one could even consider splitting methods with $a_{i}$ 's having positive real part and unconstrained complex $b_{i}$ 's.

It may be worth mentioning that the size of the arguments of the $a_{i}$ coefficients of the splitting method is a critical factor when the diffusion operator involves a complex number, for instance, an equation of the form

$$
\frac{\partial u}{\partial t}=\delta \Delta u+F(u)
$$

where $\delta$ is a complex number with $\Re(\delta)>0$. The choice $F(u)=\mu_{3} u^{3}+\mu_{2} u^{2}+\mu_{1} u+$ $\mu_{0}$ is known as the cubic Ginzburg-Landau equation FT88. In this situation, the values of the $\tilde{a}_{i}:=\arg (\delta)+\arg \left(a_{i}\right)$ determine whether the splitting method makes sense for this specific value of $\delta$. If for all $i=1, \ldots, s, \tilde{a}_{i} \in\left[-\frac{\pi}{2},+\frac{\pi}{2}\right]$, then the method is well defined. In order for the method to be applicable to such class of equations, it would make sense to try to minimize the value of $\max _{i=1, \ldots, s}\left|\arg \left(a_{i}\right)\right|$.

In this work, however, we focus on the case where the operator $A$ has a real spectrum, and thus we will only require that $\left|\arg \left(a_{i}\right)\right| \leq \pi / 2$ (i.e., $\Re\left(a_{i}\right) \geq 0$ ) while minimizing the local error coefficients. In this sense, we have observed that the coefficients of accurate splitting methods with $\Re\left(a_{i}\right) \geq 0$ tend to have also $b_{i}$ coefficients with positive real parts.

The plan of the paper is the following. In Section 2, we shall prove that if an $s$ jump construction is carried out from a basic symmetric second-order method, it is bounded to order 14 and no more and we will further justify why solving directly the system of order conditions leads to more efficient methods. In Section 3, we solve the corresponding order conditions of methods based on compositions of second-order integrators and construct several splitting methods whose coefficients have positive real part. In particular, in Subsection 3.3 we present splitting methods of orders 6 and 8, obtained as a composition scheme with Strang splitting as basic integrator. In addition, with the aim of showing that 14 is not an order barrier in general, we have built explicitly a method of order 16 as a composition of methods of order 8 , which in turn is obtained by composing the second-order Strang splitting. In Section 4 we describe the implementation of the various methods obtained in this paper and show their efficiency as compared to already available methods on two test problems. Finally, Section 5 contains some discussion and concluding remarks.

\section{AN ORDER BARRIER FOR THE $s$-JUMP CONSTRUCTION}

A simple and very fruitful technique to build high-order methods is to consider compositions of low-order ones with fractional time steps. In this way, numerical integrators of arbitrarily high order can be obtained. For splitting methods aimed to integrate problems of the form (1.1), it is necessary, however, that the coefficients have positive real part. The procedure has been carried out in CCDV09, HO09b, where composition methods up to order 14 have been constructed. We shall prove here that 14 constitutes indeed an order barrier for this kind of approach. In other words, the composition technique used in CCDV09, HO09b does not allow for the construction of methods having all of their coefficients in $\mathbb{C}_{+}:=\{z \in \mathbb{C}: \Re(z) \geq$ 
0 \} with orders strictly greater than 14 . With this goal in mind we consider the following two families of methods:

Family I. Given a method of order $p, \Phi^{[p]}(h)=\mathrm{e}^{h(A+B)}+\mathcal{O}\left(h^{p+1}\right)$, a sequence of methods of orders $p+1, p+2, \ldots$ can be defined recursively by the compositions

$$
\Phi^{[p+q]}(h)=\prod_{i=1}^{m_{q}} \Phi^{[p+q-1]}\left(\alpha_{q, i} h\right):=\Phi^{[p+q-1]}\left(\alpha_{q, 1} h\right) \cdots \Phi^{[p+q-1]}\left(\alpha_{q, m_{q}} h\right),
$$

where for all $q \geq 1$,

$$
\left(\forall 1 \leq i \leq m_{q}, \alpha_{q, i} \neq 0\right), \quad \sum_{i=1}^{m_{q}} \alpha_{q, i}=1 \quad \text { and } \quad \sum_{i=1}^{m_{q}} \alpha_{q, i}^{p+q}=0 .
$$

(Hereafter, we will interpret the product symbol from left to right). Notice that if $p+q$ is even, the second condition has only complex solutions.

Family II. Given a symmetric method of order $2 p, \tilde{\Phi}^{[2 p]}(h)$, a sequence of methods of orders $2(p+1), 2(p+2), \ldots$ can be defined recursively by the symmetric compositions

$$
\forall q \geq 1, \quad \tilde{\Phi}^{[2(p+q)]}(h)=\prod_{i=1}^{m_{q}} \tilde{\Phi}^{[2(p+q)-2]}\left(\alpha_{q, i} h\right)
$$

where $\alpha_{q, m_{q}+1-i}=\alpha_{q, i}, i=1,2, \ldots$, and for all $q \geq 1$,

$$
\left(\forall 1 \leq i \leq m_{q}, \alpha_{q, i} \neq 0\right), \quad \sum_{i=1}^{m_{q}} \alpha_{q, i}=1 \quad \text { and } \quad \sum_{i=1}^{m_{q}} \alpha_{q, i}^{2(p+q)-1}=0 .
$$

Methods of this class with real coefficients have been constructed by Creutz \& Gocksch CG89, Suzuki Suz90 and Yoshida Yos90. However, the second condition clearly indicates that at least one of the coefficients must be negative. In contrast, there exist many complex solutions with coefficients in $\mathbb{C}_{+}$.

Generally speaking, starting from $\Phi^{[1]}(h)$, the $(p+1)$-th member of Family I is of the form

$$
\Phi^{[p+1]}(h)=\prod_{i_{p}=1}^{m_{p}}\left(\prod_{i_{p-1}=1}^{m_{p-1}}\left(\cdots\left(\prod_{i_{1}=1}^{m_{1}} \Phi^{[1]}\left(\alpha_{p, i_{p}} \alpha_{p-1, i_{p-1}} \cdots \alpha_{1, i_{1}} h\right)\right) \cdots\right)\right)
$$

and has coefficients

$$
\prod_{j=1}^{p} \alpha_{j, i_{j}} \quad 1 \leq i_{1} \leq m_{1}, \ldots, 1 \leq i_{p} \leq m_{p} .
$$

A similar expression holds, of course, for methods of Family II, starting from $\tilde{\Phi}^{[2]}(h)$. Symmetric compositions for the cases $m_{1}=m_{2}=\cdots=m_{p}=3$ and $m_{1}=m_{2}=\cdots=m_{p}=4$, correspond to the triple and quadruple jump techniques, respectively.

Lemma 2.1. Let $\Phi(h)$ be a consistent method (i.e., a method of order $p \geq 1$ ) and assume that the method

$$
\Psi(h)=\prod_{i=1}^{l} \Phi\left(\alpha_{i} h\right)
$$



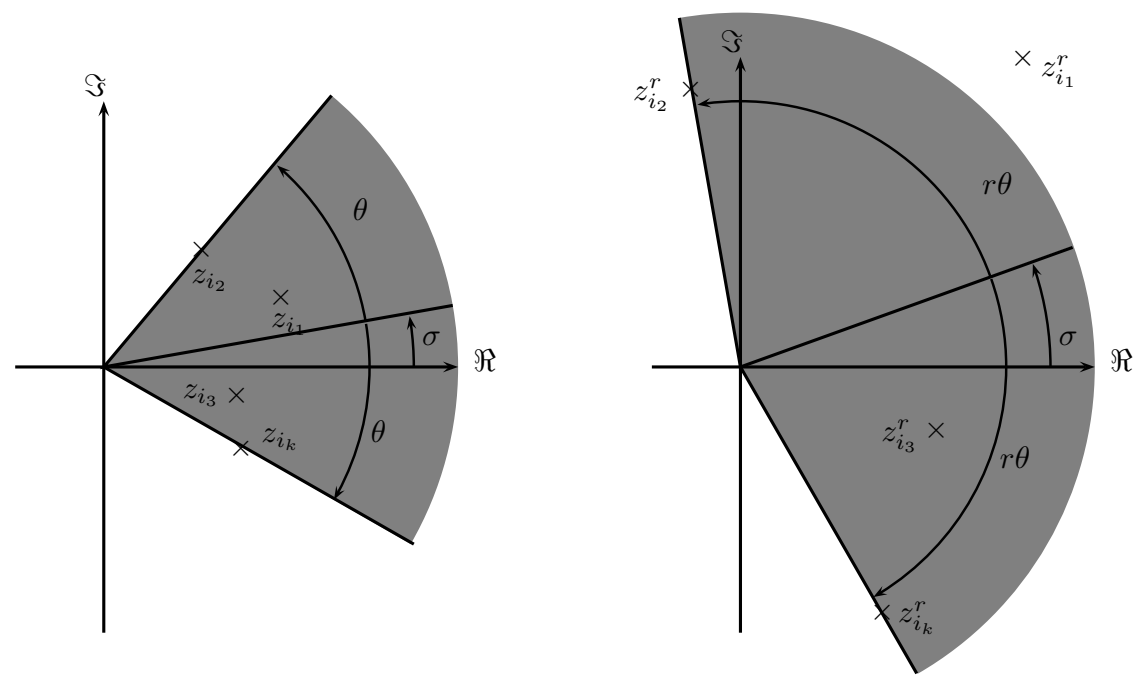

Figure 1. The enveloping sectors of $\left\{z_{1}, \ldots, z_{k}\right\} \subset \mathbb{C}_{+}$and of $\left\{z_{1}^{r}, \ldots, z_{k}^{r}\right\} \subset \mathbb{C}($ for $r=2)$.

is also consistent (i.e., $\sum_{i} \alpha_{i}=1$ ). If there exists $k, 1 \leq k \leq l$, such that $\Re\left(\alpha_{k}\right)<0$, then any consistent method of the form

$$
\prod_{j=1}^{m} \Psi\left(\beta_{j} h\right)=\prod_{j=1}^{m}\left(\prod_{i=1}^{l} \Phi\left(\beta_{j} \alpha_{i} h\right)\right)
$$

has at least one coefficient $\beta_{j} \alpha_{k}, 1 \leq j \leq m$, such that $\Re\left(\beta_{j} \alpha_{k}\right)<0$.

Proof. By consistency one has $\sum_{j=1}^{m} \beta_{j}=1$, so that

$$
\sum_{j=1}^{m} \Re\left(\beta_{j} \alpha_{k}\right)=\Re\left(\sum_{j=1}^{m} \beta_{j} \alpha_{k}\right)=\Re\left(\alpha_{k} \sum_{j=1}^{m} \beta_{j}\right)=\Re\left(\alpha_{k}\right)<0 .
$$

This implies the statement.

Lemma 2.2. For $k \geq 2$ and $r \geq 2$, consider $\left(z_{1}, \ldots, z_{k}\right) \in\left(\mathbb{C}_{+}\right)^{k}$ such that $\sum_{i=1}^{k} z_{i}^{r}=0, z_{i} \neq 0$ for $i=1, \ldots, k$. Then we have

$$
\max _{i=1, \ldots, k} \operatorname{Arg}\left(z_{i}\right)-\min _{i=1, \ldots, k} \operatorname{Arg}\left(z_{i}\right) \geq \frac{\pi}{r} .
$$

Proof. All the $z_{i}$ 's belong to the sector $K_{\sigma}(\theta)=\{z \in \mathbb{C}:|\sigma-\operatorname{Arg}(z)| \leq \theta\}$ with

and

$$
\sigma=\frac{1}{2}\left(\max _{i=1, \ldots, k} \operatorname{Arg}\left(z_{i}\right)+\min _{i=1, \ldots, k} \operatorname{Arg}\left(z_{i}\right)\right)
$$

$$
\theta=\frac{1}{2}\left(\max _{i=1, \ldots, k} \operatorname{Arg}\left(z_{i}\right)-\min _{i=1, \ldots, k} \operatorname{Arg}\left(z_{i}\right)\right),
$$

where Arg is the principal value of the argument (see the left picture of Figure 11). Now, assume that $\theta<\frac{\pi}{2 r}$. Then, all the $z_{i}^{r}$ 's belong to $K_{r \sigma}(r \theta)$, which, since 
$r \theta<\frac{\pi}{2}$, is a convex set. This implies that $\sum_{i=1}^{k} z_{i}^{r}$ also belongs to $K_{r \sigma}(r \theta)$ (see the right picture of Figure 1). The inequality $r \theta<\frac{\pi}{2}$ being strict and the $z_{i}$ 's non-zero, we have furthermore $\sum_{i=1}^{k} z_{i}^{r} \neq 0$, which contradicts the assumption. The result follows.

Theorem 2.3. (i) Starting from a first-order method $\Phi^{[1]}(h)$, all methods $\Phi^{[p+1]}(h)$ of order $p+1, p=3,4, \ldots$ from Family I have at least one coefficient with negative real part.

(ii) Starting from a second-order symmetric method $\tilde{\Phi}^{[2]}(h)$, all methods $\tilde{\Phi}^{[2 p+2]}(h)$ of order $2 p+2, p=7,8, \ldots$ from Family II have at least one coefficient with negative real part.

Proof. We prove at once the two assertions. We first notice that, according to Lemma 2.1, if method $\Phi^{[p]}(h)$ of Family I (respectively, method $\tilde{\Phi}^{[2 p]}(h)$ of Family II), has a coefficient with negative real part, then all subsequent methods $\Phi^{[p+q]}(h)$, $q \geq 1$, of Family I (respectively, methods $\tilde{\Phi}^{[2(p+q)]}(h)$ of Family II), also have a coefficient with negative real part. Hence, we assume that all methods $\Phi^{[q+1]}(h)$, $q=1, \ldots, p$ from Family I (respectively, all methods $\tilde{\Phi}^{[2 q+2]}(h)$ of Family II), have all their coefficients in $\mathbb{C}_{+}$. Using Lemma 2.2 we have

$$
\forall q=1, \ldots, p, \quad \max _{i=1, \ldots, m_{q}} \operatorname{Arg}\left(\alpha_{q, i}\right)-\min _{i=1, \ldots, m_{q}} \operatorname{Arg}\left(\alpha_{q, i}\right) \geq \frac{\pi}{q+1},
$$

respectively,

$$
\forall q=1, \ldots, p, \quad \max _{i=1, \ldots, m_{q}} \operatorname{Arg}\left(\alpha_{q, i}\right)-\min _{i=1, \ldots, m_{q}} \operatorname{Arg}\left(\alpha_{q, i}\right) \geq \frac{\pi}{2 q+1},
$$

so that

$$
\begin{aligned}
& \max _{1 \leq i_{1} \leq m_{1}, \ldots, 1 \leq i_{p} \leq m_{p}} \operatorname{Arg}\left(\prod_{j=1}^{p} \alpha_{j, i_{j}}\right)-\min _{1 \leq i_{1} \leq m_{1}, \ldots, 1 \leq i_{p} \leq m_{p}} \operatorname{Arg}\left(\prod_{j=1}^{p} \alpha_{j, i_{j}}\right), \\
& \geq \frac{\pi}{2}+\cdots+\frac{\pi}{p+1}
\end{aligned}
$$

respectively,

$$
\begin{aligned}
& \max _{1 \leq i_{1} \leq m_{1}, \ldots, 1 \leq i_{p} \leq m_{p}} \operatorname{Arg}\left(\prod_{j=1}^{p} \alpha_{j, i_{j}}\right)-\min _{1 \leq i_{1} \leq m_{1}, \ldots, 1 \leq i_{p} \leq m_{p}} \operatorname{Arg}\left(\prod_{j=1}^{p} \alpha_{j, i_{j}}\right) \\
& \geq \frac{\pi}{3}+\cdots+\frac{\pi}{2 p+1} .
\end{aligned}
$$

Now, since $\frac{1}{2}+\frac{1}{3}+\frac{1}{4}>1, p=3$ in the first case and thus the first statement follows. For Family II, since $\frac{1}{3}+\frac{1}{5}+\cdots+\frac{1}{15}>1$, then $p=7$, thus leading to the second statement.

Remark 2.4. No method of Family I with coefficients in $\mathbb{C}_{+}$can have an order strictly greater than 3 . Such methods of order 3 have been constructed in HO09b. Similarly, no method of Family II with coefficients in $\mathbb{C}_{+}$can have an order strictly greater than 14. Such methods with orders up to 14 have been constructed in CCDV09, HO09b]. For instance, let us consider the quadruple jump composition

$$
\tilde{\Phi}^{[2 p+2]}(h)=\tilde{\Phi}^{[2 p]}\left(\alpha_{p, 1} h\right) \tilde{\Phi}^{[2 p]}\left(\alpha_{p, 2} h\right) \tilde{\Phi}^{[2 p]}\left(\alpha_{p, 2} h\right) \tilde{\Phi}^{[2 p]}\left(\alpha_{p, 1} h\right),
$$


where $\alpha_{p, 1}+\alpha_{p, 2}=1 / 2$ and $\alpha_{p, 1}^{2 p+1}+\alpha_{p, 2}^{2 p+1}=0$. This system has $p$ solutions (and their complex conjugate). The solution with minimal argument is, as noticed in CCDV09, HO09b,

$$
\alpha_{p, 1}=\frac{1}{4}\left(1+i \frac{\sin \left(\frac{\pi}{2 p+1}\right)}{1+\cos \left(\frac{\pi}{2 p+1}\right)}\right), \quad \alpha_{p, 2}=\bar{\alpha}_{p, 1}
$$

(and its complex conjugate). It is straightforward to verify that $\arg \left(\alpha_{p, 1}\right)=\frac{\pi}{2(2 p+1)}$ and $\arg \left(\alpha_{p, 2}\right)=-\frac{\pi}{2(2 p+1)}$, so that

$$
\arg \left(\alpha_{p, 1}\right)-\arg \left(\alpha_{p, 2}\right)=\frac{\pi}{2 p+1} .
$$

Comparing with the proof of Theorem 2.3. we observe that the bounds obtained there are sharp since a family of methods do exist, satisfying the equality.

Remark 2.5. It is, however, possible to construct a composition method with all coefficients having positive real part of order strictly greater than 14 directly from a symmetric second-order method. For example, in Subsection 3.3 we present a new method of sixteenth-order built as

$$
\Phi^{[16]}(h)=\prod_{i=1}^{21} \Phi^{[8]}\left(\alpha_{i} h\right), \quad \text { with } \quad \Phi^{[8]}(h)=\prod_{j=1}^{15} \Phi^{[2]}\left(\beta_{j} h\right)
$$

and the coefficients satisfying $\Re\left(\alpha_{i} \beta_{j}\right)>0$ for all $i=1, \ldots, 21, j=1, \ldots, 15$, with $\alpha_{22-i}=\alpha_{i}, \beta_{16-j}=\beta_{j}, i, j=1,2, \ldots$ Here, $\Phi^{[8]}(h)$ is a symmetric composition of symmetric second-order methods, but it is not a composition of methods of order 4 or 6 , and similarly for $\Phi^{[16]}(h)$, which is not a composition of methods of orders 10,12 or 14 .

Theorem 2.6. Splitting methods of the class (1.2) with $\Re\left(a_{i}\right) \geq 0$ exist at least up to order 44.

Proof. In CCDV09, a fourth-order method was obtained with $a_{i} \in \mathbb{R}^{+}$. In a similar way, we have also built a sixth-order scheme with $a_{i} \in \mathbb{R}^{+}$(whose coefficients can be found at http://www.gicas.uji.es/Research/splitting-complex.html). Using this as the basic method in the quadruple jump (2.7) with coefficients chosen with the minimal argument and, since

$$
\frac{1}{7}+\cdots+\frac{1}{43}<1<\frac{1}{7}+\cdots+\frac{1}{45}
$$

we conclude that all methods obtained up to order 44 will satisfy that $\Re\left(a_{i}\right) \geq 0$.

Remark 2.7. The question of the existence of splitting schemes at any order with $\Re\left(a_{i}\right) \geq 0$ still remains open.

\section{Splitting methods With ALL COEFFicients HaVing POSITIVe REAL PART}

3.1. Order conditions and leading terms of local error. We have seen that the composition technique to construct high-order methods inevitably leads to an order barrier. In addition, the resulting methods require a large number of evaluations (i.e., $3^{n-1}$ or $4^{n-1}$ evaluations to get order $2 n$ using the triple or quadruple jump, respectively) and usually have large truncation errors. In this section we 
show that, as with real coefficients, it is indeed possible to build very efficient highorder splitting methods whose coefficients have positive real part by solving directly the order conditions necessary to achieve a given order $p$. These are, roughly speaking, large systems of polynomial equations in the coefficients $a_{i}, b_{i}$ of the method (1.2), arising by requiring that the formal expansion of the method satisfies (1.5) for arbitrary non-commuting operators $A$ and $B$.

Different (but equivalent) formulations of such order conditions exist in the literature HLW06. Among them, the one using Lyndon multi-indices is particularly appealing. It was first introduced in CM09] (see also [BCM08]) and can be considered as a variant of the classical treatment obtained in MSS99.

This analysis shows that the number of order conditions for general splitting methods of the form (1.2) grows very rapidly with the order $p$, even when one considers only symmetric methods. For instance, there are 26 independent eighthorder conditions and 82 tenth-order conditions for a consistent symmetric splitting method. It makes sense, then, to examine alternatives to achieve order higher than six. This can be accomplished by taking compositions of a basic symmetric method of even-order. In particular, if we consider any of the two versions of Strang splitting (1.4) as the basic method $S(h)$, then, for each $\gamma=\left(\gamma_{1}, \ldots, \gamma_{s}\right) \in \mathbb{C}^{s}$,

$$
\Psi(h)=S\left(\gamma_{1} h\right) \cdots S\left(\gamma_{s} h\right)
$$

will be a new splitting method of the form (1.2). Now the consistency condition reads

$$
\gamma_{1}+\cdots+\gamma_{s}=1
$$

As for the additional conditions to attain order $p$, these can be obtained by generalizing the treatment done in MSS99. Splitting methods with very high-order can be constructed as (3.1) by considering as basic method $S(h)$ a symmetric method of even order $2 q>2$. In that case, it can be shown that the corresponding number of order conditions is considerably reduced with respect to (1.2). Thus, for instance, if $S(h)$ is a symmetric splitting method of order eight, a method (3.1) satisfying the consistency condition (3.2) and the symmetry condition

$$
\gamma_{s-j+1}=\gamma_{j}, \quad 1 \leq j \leq s
$$

only needs to satisfy 10 additional conditions to attain order sixteen.

3.2. Leading term of the local error. To construct splitting methods of a given order $p$ within a family of schemes, we choose the number $s$ of stages in such a way that the number of unknowns equals the number of order conditions, so that one typically has a finite number of isolated (real or complex) solutions, each of them leading to a different splitting method. Among them, we will be interested in methods such that, either $a_{i} \geq 0$ (and each $b_{i}$ are arbitrary complex numbers) or $\Re\left(a_{i}\right) \geq 0$ and $\Re\left(b_{i}\right) \geq 0$. The relevant question at this point is how to choose the "best" solution in the set of all solutions satisfying the required conditions. It is generally accepted that good splitting methods must have small coefficients $a_{i}, b_{i}$. Methods with large coefficients tend to show bad performance in general, which is particularly true when relatively long time-steps $h$ are used. In addition, as mentioned in the introduction, when applying splitting methods to the class of problems considered here, the arguments of the complex coefficients $a_{i}, b_{i}$ must also be taken into account. 
In order to choose the best scheme among two methods with coefficients of similar size included in sectors with similar angle, we analyze the leading term of the local error of the splitting method. If (1.2) is of order $p$, then we formally have that

$$
\begin{aligned}
\Psi(h)-\mathrm{e}^{h(A+B)} & =h^{p+1} E_{p+1}+\mathcal{O}\left(h^{p+1}\right), \\
E_{p+1} & :=\sum_{i_{1}+\cdots+i_{2 m}=p+1} v_{i_{1}, \ldots, i_{2 m}}(\gamma) A^{i_{1}} B^{i_{2}} \ldots A^{i_{2 m-1}} B^{i_{2 m}},
\end{aligned}
$$

where $\gamma=\left(\gamma_{1}, \ldots, \gamma_{s}\right)$ is given in terms of the original coefficients $a_{i}, b_{i}$ of the integrator by

$$
a_{j}=\gamma_{j}, \quad b_{j-1}=\frac{\gamma_{j-1}+\gamma_{j}}{2}
$$

$\left(\gamma_{0}=\gamma_{s+1}=0\right)$ and each $v_{i_{1}, \ldots, i_{2 m}}(\gamma)$ is a linear combination of polynomials in $\gamma$ BCM08.

Incorporating that into the results in $\mathrm{HO} 09 \mathrm{~b}$, it can be shown that, if the smoothness assumption stated in the introduction is increased from $p+1$ to $p+2$, then for sufficiently small $h$, the local error is dominated by $\left\|h^{p+1} E_{p+1} u_{0}\right\|$.

Our strategy to select a suitable method among all possible choices is then the following: first choose a subset of solutions with reasonably small maximum norm of the coefficient vector $\left(\gamma_{1}, \ldots, \gamma_{s}\right)$, and then, among them, choose the one that minimizes the norm

$$
\sum_{i_{1}+\cdots+i_{2 m}=p+1}\left|v_{i_{1} \cdots i_{2 m}}(\gamma)\right|
$$

of the coefficients of the leading term of the local error. This seems reasonable if one is interested in choosing a splitting method that works fine for arbitrary operators $A$ and $B$ satisfying the semi-group and smoothness conditions mentioned in the introduction. Of course, this does not guarantee that a method with a smaller value of (3.4) will be more precise for any $A$ and $B$ than another method with a larger value of (3.4). However, we have observed in practice when solving the order conditions of different families of splitting methods, that the solution that minimizes (3.4) tend to have smaller values of most (or even all) coefficients $\left|v_{i_{1} \cdots i_{2 m}}(\gamma)\right|$ when compared to a solution having a larger norm (3.4) of the coefficients of the leading term of the local error.

When $A$ and $B$ are operators in a real Banach space $X$, then it makes sense to compute the approximations $u_{n}=u\left(t_{n}\right)$, as $u_{n}=\Re(\Psi(h)) u_{n-1}$. In that case, the argument above holds with $\Psi(h)$ replaced by $\hat{\Psi}(h)=\Re(\Psi(h))$ and the local error coefficients $v_{i_{1}, \ldots, i_{2 m}}(\gamma)$ replaced by $\Re\left(v_{i_{1}, \ldots, i_{2 m}}(\gamma)\right)$. In that case, (3.4) should be replaced by

$$
\sum_{i_{1}+\cdots+i_{2 m}=p+1}\left|\Re\left(v_{i_{1}, \ldots, i_{2 m}}(\gamma)\right)\right|
$$

as a general measure of leading term of the local error. 


\subsection{High-order splitting methods obtained as a composition of simpler methods.}

Order 6. We first consider sixth-order symmetric splitting methods obtained as a composition (3.1) of the Strang splitting (1.4) as the basic method. In this case the coefficients $\gamma_{i}$ must satisfy three order conditions, in addition to the symmetry (3.3) and consistency requirements, to achieve order six. We thus take $s=7$, so that we have three equations and three unknowns. Such a system of polynomial equations has 39 solutions in the complex domain (three real solutions among them), 12 of them giving a splitting method with coefficients of positive real part. According to the criteria described in Subsection 3.2, we arrive at the scheme

$$
\begin{aligned}
\gamma_{1}=\gamma_{7} & =0.116900037554661284389+0.043428254616060341762 i \\
\gamma_{2}=\gamma_{6} & =0.12955910128208826275-0.12398961218809259330 i \\
\gamma_{3}=\gamma_{5} & =0.18653249281213381780+0.00310743071007267534 i \\
\gamma_{4} & =0.134016736702233270122+0.154907853723919152396 i
\end{aligned}
$$

This method turns out to correspond to one of those obtained by Chambers (see Table 4 in Cha03]).

Order 8. For consistent symmetric methods (3.1) of order eight, we have seven order conditions. By taking $s=15$ stages, one ends up with a system of seven polynomial equations and seven unknowns. We have performed an extensive numerical search of solutions with small norm, finding 326 complex solutions. Among them, 162 lead to splitting methods whose coefficients possess positive real part. The best method, according to the criteria established in Subsection 3.2, is

$$
\text { (3.7) } \begin{aligned}
\gamma_{1}=\gamma_{15} & =0.053475778387618596606+0.006169356340079532510 i, \\
\gamma_{2}=\gamma_{14} & =0.041276342845804256647-0.069948574390707814951 i, \\
\gamma_{3}=\gamma_{13} & =0.086533558604675710289-0.023112501636914874384 i, \\
\gamma_{4}=\gamma_{12} & =0.079648855663021043369+0.049780495455654338124 i, \\
\gamma_{5}=\gamma_{11} & =0.069981052846323122899-0.052623937841590541286 i, \\
\gamma_{6}=\gamma_{10} & =0.087295480759955219242+0.010035268644688733950 i, \\
\gamma_{7}=\gamma_{9} & =0.042812886419632082126+0.076059456458843523862 i, \\
\gamma_{8} & =0.077952088945939937643+0.007280873939894204350 i .
\end{aligned}
$$

Order 16. Motivated by the results in Section 2 , we have also constructed a splitting method of order 16. Our aim, rather than proposing a very efficient scheme, is to show that the barrier of order 14 existing for methods built by applying the recursive composition technique starting from order two (Family II) does not apply in general.

The construction procedure can be summarized as follows. We consider a consistent symmetric composition of the form (3.1), where now the basic method $S(h)$ is any symmetric eighth-order scheme. Under such conditions, ten order conditions must be satisfied to achieve order 16 . We accordingly take $s=21$, so that we have ten polynomial equations with ten unknowns. We have performed an extensive numerical search of solutions with relatively small norm, finding 70 complex solutions with positive real part. Combined with the 162 methods of order eight, this leads 
to 11340 different sixteenth-order splitting methods with $s=315$ stages. Among them, only 324 give rise to splitting methods with coefficients of positive real part. The coefficients of the method that we have determined as optimal can be found at http://www.gicas.uji.es/Research/splitting-complex.html.

Remark 3.1. Notice that, whereas for the sixth-order method (3.6) one has

$$
\max _{i=1, \ldots, 7} \operatorname{Arg}\left(\gamma_{i}\right)-\min _{i=1, \ldots, 7} \operatorname{Arg}\left(\gamma_{i}\right) \approx 1.621<\frac{\pi}{3}+\frac{\pi}{5}
$$

and then the coefficients $\gamma_{i}$ are distributed in a narrower sector than for triple or quadruple jump methods, for the eighth-order method (3.7) one has

$$
\max _{i=1, \ldots, 15} \operatorname{Arg}\left(\gamma_{i}\right)-\min _{i=1, \ldots, 15} \operatorname{Arg}\left(\gamma_{i}\right) \approx 2.5997>\frac{\pi}{3}+\frac{\pi}{5}+\frac{\pi}{7} .
$$

This method, whereas being the most efficient, is not the appropriate one to be used as basic scheme to build higher-order methods by composition. The previous sixteenth-order integrator has been built starting with another eighth-order method whose coefficients are placed in a narrower sector.

\section{NUMERICAL TESTS}

For our numerical experiments, we consider two different test problems: a linear reaction-diffusion equation, and the semi-linear Fisher's equation, both with periodic boundary conditions in space. It should be mentioned here that this last case is not covered by the theoretical framework summarized in the Introduction. For each case, we detail the experimental setting and collect the results achieved by the different schemes. Our main purpose here is just to illustrate the performance of the new splitting methods to carry out the time integration as compared with those constructed by using the Yoshida-Suzuki triple jump composition technique for both examples. Notice that, in this sense, the particular scheme used to discretize in space is irrelevant. For that reason, and to keep the treatment as simple as possible, we have applied a simple second-order finite difference scheme in space.

The numerical approximations $u_{n}$ obtained by each method $\Psi(h)$ are computed as $u_{n}=\Re(\Psi(h)) u_{n-1}$. In other words, we project on the real axis after completing each time step.

4.1. A linear parabolic equation. Our first test-problem is the scalar equation in one-dimension

$$
\frac{\partial u(x, t)}{\partial t}=\alpha \Delta u(x, t)+V(x, t) u(x, t), \quad u(x, 0)=u_{0}(x)
$$

with $u_{0}(x)=\sin (2 \pi x)$ and periodic boundary conditions in the space domain $[0,1]$. We take $\alpha=\frac{1}{4}, V(x, t)=3+\sin (2 \pi x)$ and discretize in space

$$
x_{j}=j(\delta x), \quad j=1, \ldots, N \quad \text { with } \quad \delta x=1 / N,
$$

thus arriving at the differential equation

$$
\frac{d U}{d t}=\alpha A U+B U
$$


where $U=\left(u_{1}, \ldots, u_{N}\right) \in \mathbb{R}^{N}$. The Laplacian $\Delta$ has been approximated by the matrix $A$ of size $N \times N$ given by

$$
A=\frac{1}{(\delta x)^{2}}\left(\begin{array}{ccccc}
-2 & 1 & & & 1 \\
1 & -2 & 1 & & \\
& 1 & -2 & 1 & \\
& & \ddots & \ddots & \ddots \\
1 & & & 1 & -2
\end{array}\right),
$$

and $B=\operatorname{diag}\left(V\left(x_{1}\right), \ldots, V\left(x_{N}\right)\right)$. We take $N=100$ points and compare different composition methods by computing the corresponding approximate solution on the time interval $[0,1]$. In particular, we consider the following schemes:

- Strang: The second-order symmetric Strang splitting method (1.4);

- (TJ6): The sixth-order triple jump method (CCDV09, Proposition 2.1) based on Strang's second-order method;

- (TJ6A): The sixth-order triple jump method (CCDV09, Proposition 2.2) based on Strang's second-order method;

- (TJ8A): The eighth-order triple jump method ([CCDV09, Proposition 2.2) based on Strang's second-order method;

- (P6S7): The sixth-order method (3.6);

- (P8S15): The eighth-order method (3.7).

We compute the error of the numerical solution at time $t=1$ (in the 2-norm) as a function of the number of evaluations of the basic method (the Strang splitting) and represent the outcome in Figure 2, In the left panel we collect the results achieved by the Strang splitting and the previous sixth-order composition methods, whereas the right panel corresponds to eighth-order methods. We have also included, for reference, the curve obtained by (P6S7).

The relative cost (w.r.t. Strang) of a method composed of $s$ steps is approximated by $4 s$, where the factor 4 stands here for an average ratio between the cost of complex arithmetic compared to real arithmetic. A remarkable outcome of these experiments is that methods (P6S7) and (P8S15) outperform Strang's splitting (and actually all other methods tested here) even for low tolerances. Scheme (P8S15), in particular, proves to be the most efficient in the whole range explored. The gain with respect to triple jump methods is also significant and completely support the approach followed here.

4.2. The semi-linear reaction-diffusion equation of Fisher. Our second testproblem is the scalar equation in one-dimension,

$$
\frac{\partial u(x, t)}{\partial t}=\Delta u(x, t)+F(u(x, t)), \quad u(x, 0)=u_{0}(x),
$$

with periodic boundary conditions in the space domain $[0,1]$. We take, in particular, Fisher's potential

$$
F(u)=u(1-u) .
$$

The splitting considered here corresponds to solving, on the one hand, the linear equation with the operator $A$ being the Laplacian, and on the other hand, the nonlinear ordinary differential equation

$$
\frac{\partial u(x, t)}{\partial t}=u(x, t)(1-u(x, t))
$$



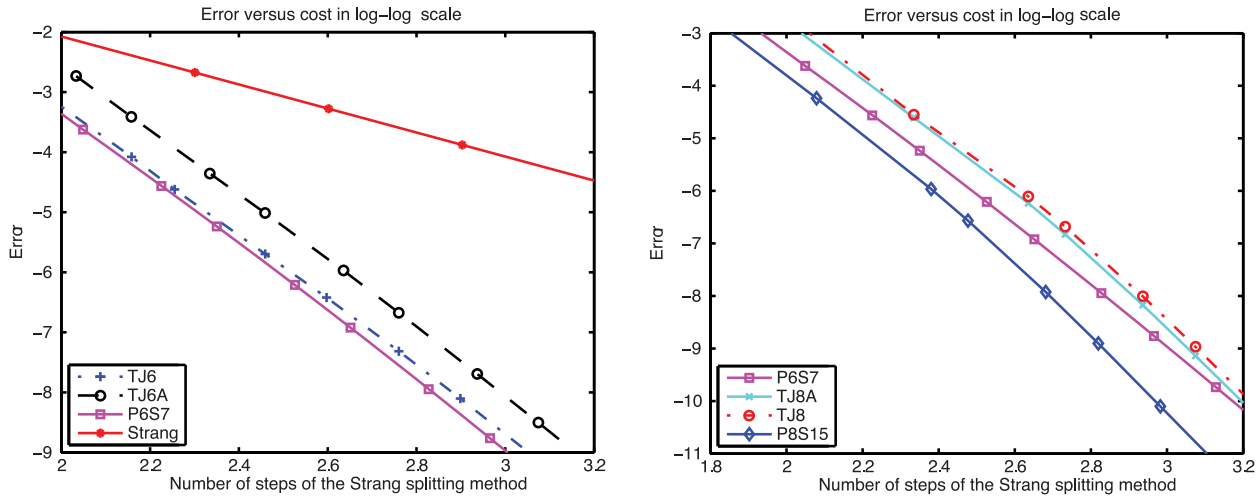

FiguRE 2. Error versus number of steps for the linear reactiondiffusion equation (4.1).
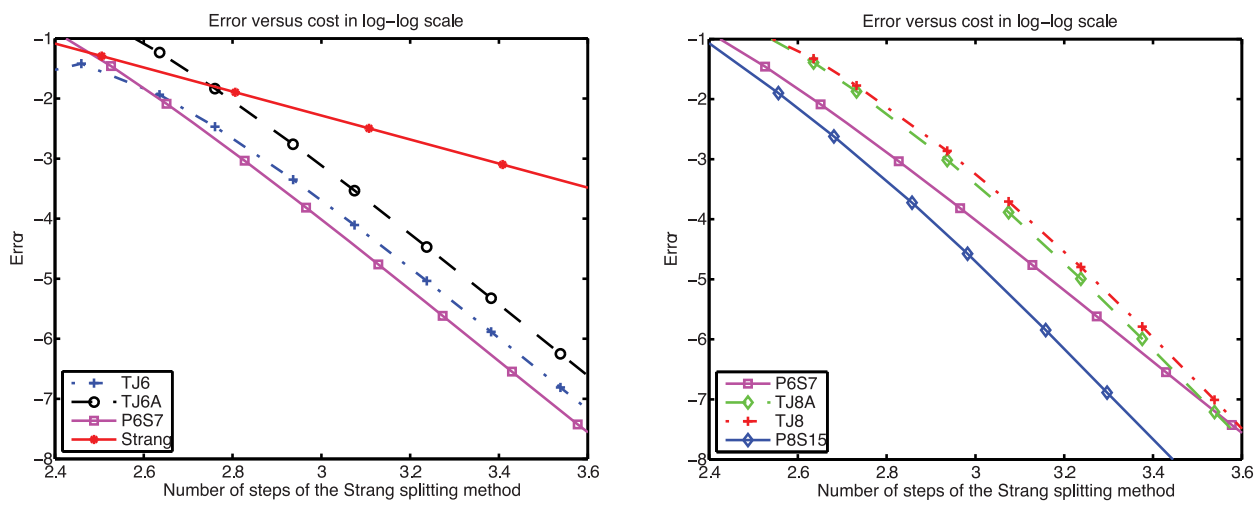

FiguRE 3. Error versus number of steps for the semi-linear reaction-diffusion equation (4.3).

with initial condition

$$
u(x, 0)=u_{0}(x) .
$$

Note that it can be solved analytically as

$$
u(x, t))=u_{0}(x)+u_{0}(x)\left(1-u_{0}(x)\right) \frac{\left(\mathrm{e}^{t}-1\right)}{1+u_{0}(x)\left(\mathrm{e}^{t}-1\right)},
$$

which is well defined for small complex time $t$. We proceed in the same way as for the previous linear case, starting with $u_{0}(x)=\sin (2 \pi x)$. After discretization in space, we arrive at the differential equation

$$
\frac{d U}{d t}=A U+F(U)
$$

where $U=\left(u_{1}, \ldots, u_{N}\right) \in \mathbb{R}^{N}$ and $F(U)$ is now defined by

$$
F(U)=\left(u_{1}\left(1-u_{1}\right), \ldots, u_{N}\left(1-u_{N}\right)\right) .
$$

We choose $N=100$ and compute the error (in the 2-norm) at the final time $t=1$ by applying the same composition methods as in the linear case. The results are collected in Figure 3, where identical notation has been used. Notice that, strictly 
speaking, the theoretical framework upon which our strategy is based does not cover this nonlinear problem. Nevertheless, the results achieved are largely similar to the linear case. In particular, the new eighth-order composition method is the most efficient even for moderate tolerances.

\section{Concluding Remarks}

Splitting methods with real coefficients for the numerical integration of differential equations of order higher than two have necessarily some negative coefficients. This feature does not suppose any special impediment when the differential equation evolves in a group, but may be unacceptable when it is defined in a semi-group, as is the case with the evolution partial differential equations considered in this paper. One way to get around this fundamental difficulty is to consider splitting schemes with complex coefficients having positive real part. This has been recently proposed for diffusion equations in [CCDV09, HO09b. Splitting and composition methods with complex coefficients have been considered in different contexts in the literature (see [BCM10] and references therein).

In CCDV09, HO09b, splitting methods up to order 14 with complex coefficients with non-negative real part have been recursively constructed either by the socalled triple-jump compositions or by the quadruple-jump compositions, starting from the symmetric second-order Strang splitting. In this work we prove that there exists indeed an order barrier of 14 for methods constructed in this way. More generally, we show that no method of order higher than 14 with coefficients having non-negative real part can be constructed by sequential $s$-jump compositions starting from a symmetric method of order 2. We further show, by explicitly obtaining methods of order 16 (as the composition of a basic symmetric method of order 8), that this order barrier does not apply for general composition methods (non-necessarily constructed by recursive applications of $s$-jump compositions) with complex coefficients with non-negative real part.

In addition to this order barrier, another drawback of methods resulting from applying the $s$-jump composition procedure is that for high orders they require a larger number of stages (i.e., number of compositions of the basic symmetric second order method) than methods obtained by directly solving the order conditions with the minimal number of stages. For instance, methods of order 6 (respectively, 8) obtained with triple jump compositions need 9 (resp. 27) compositions of the basic second-order method, whereas, as we show in the present work, methods of order 6 (resp. 8) can be constructed (by directly solving for the required order conditions) with 7 (resp. 15) stages. An analysis of the local error coefficients supported by numerical tests shows that the methods proposed here are more efficient than those obtained in CCDV09, HO09b by applying the recursive triple jump and quadruple jump constructions. An additional requirement when choosing a given method is that the arguments of the complex coefficients of the scheme also have to be taken into account. This constitutes a critical point for evolution equations where one of the operators (say, $A$ ) has non-real eigenvalues in the right-hand side of the complex plane, as occurs, in particular, with the complex Ginzburg-Landau equation (1.6). In such a case, splitting methods of the form (1.2) where one of the two sets of coefficients $a_{i}$ or $b_{i}$ is entirely contained in the positive real axis, whereas the other set included in the right-hand side of the complex planes are particularly well suited. Such splitting methods cannot be constructed as composition methods 
with the Strang splitting as the basic method, so that a separate study is required to get the most efficient schemes within this class.

Based on the theoretical framework worked out in [HO09a], the integrators proposed here can be applied to the numerical integration of linear evolution equations involving unbounded operators in an infinite dimensional space, like linear diffusion equations. As a matter of fact, although the theory developed in HO09a does not cover the generalization to semi-linear evolution equations, we have also included in our numerical tests a system of ODEs obtained from semi-linear evolution equations with a certain space discretization. All the numerical tests carried out with periodic boundary conditions show a considerable improvement in efficiency of our new methods with respect to existing splitting schemes. A remarkable feature of the new eighth-order composition method when applied to both the linear and semilinear diffusion examples is that it is more efficient than all the other integrators of order $p \leq 8$ in the whole range of tolerances explored when periodic boundary conditions are considered.

Concerning other (e.g., Dirichlet and Neumann) boundary conditions, the experiments carried out in [HO09b] for linear problems with methods obtained by applying the triple and quadruple jump technique show the existence of an order reduction phenomenon. Its origin is attributed by the authors of $\mathrm{HO} 09 \mathrm{~b}$ ] to the fact that Condition 2 in the introduction is not generally satisfied in this setting. In other words, terms of the form $E_{p+1} \mathrm{e}^{t(A+B)}$ in (1.5) are not uniformly bounded on the interval $[0, T]$ for some $T>0$. As a consequence, the classical convergence order is no longer guaranteed in that case. This order reduction is also present, of course, when the methods introduced in this paper are applied not only to linear but also semi-linear problems. Nevertheless, as pointed out in HO09b, splitting schemes of high-order involving complex coefficients may still be advantageous in comparison with, say, Strang splitting when applied to linear parabolic problems with Dirichlet or Neumann boundary conditions, as they often lead to smaller errors and the order reduction is somehow confined to a neighborhood of the boundary. The situation, in our opinion, calls for a detailed study of the effect of boundary conditions other than the periodic case in the global efficiency of splitting methods with complex coefficients and the analysis of their applicability for more general parabolic problems than those considered in this paper.

\section{REFERENCES}

[BC05] S. Blanes and F. Casas. On the necessity of negative coefficients for operator splitting schemes of order higher than two. Appl. Num. Math., 54:23-37, 2005. MR2134093 (2006b:65085)

[BCM08] S. Blanes, F. Casas, and A. Murua. Splitting and composition methods in the numerical integration of differential equations. Bol. Soc. Esp. Mat. Apl., 45:87-143, 2008. MR2477860 (2010c:65250)

[BCM10] S. Blanes, F. Casas, and A. Murua. Splitting methods with complex coefficients. Bol. Soc. Esp. Mat. Apl., 50:47-61, 2010. MR2664321 (2011e:65101)

[CCDV09] F. Castella, P. Chartier, S. Descombes, and G. Vilmart. Splitting methods with complex times for parabolic equations. BIT Numerical Analysis, 49:487-508, 2009. MR 2545817 (2010k:65098)

[CG89] M. Creutz and A. Gocksch. Higher-order hybrid Monte Carlo algorithms. Phys. Rev. Lett., 63:9-12, 1989. MR.1001905 (90e:82005)

[Cha03] J. E. Chambers. Symplectic integrators with complex time steps. Astron. J., 126:1119$1126,2003$. 
[CM09] P. Chartier and A. Murua. An algebraic theory of order. M2AN Math. Model. Numer. Anal., 43:607-630, 2009. MR2542867 (2010h:65107)

[DS02] S. Descombes and M. Schatzman. Strang's formula for holomorphic semi-groups. J. Math. Pures Appl., 81:93-114, 2002. MR1994884 (2005g:35008)

[For89] E. Forest. Canonical integrators as tracking codes. AIP Conference Proceedings, 184:1106-1136, 1989.

[FT88] S. Fauve and O. Thual. Localized structures generated by subcritical instabilities. $J$. Phys. France, 49:1829-1833, 1988.

[GK96] D. Goldman and T. J. Kaper. $n$ th-order operator splitting schemes and nonreversible systems. SIAM J. Numer. Anal., 33:349-367, 1996. MR.1377257 (97a:65063)

[HLW06] E. Hairer, C. Lubich, and G. Wanner. Geometric Numerical Integration. StructurePreserving Algorithms for Ordinary Differential Equations, Second edition. Springer Series in Computational Mathematics 31. Springer, Berlin, 2006. MR2221614 (2006m:65006)

[HO09a] E. Hansen and A. Ostermann. Exponential splitting for unbounded operators. Math. Comp., 78:1485-1496, 2009. MR2501059 (2011b:65079)

[HO09b] E. Hansen and A. Ostermann. High order splitting methods for analytic semigroups exist. BIT Numerical Analysis, 49:527-542, 2009. MR2545819 (2010k:65100)

[HKLR10] H. Holden, K.H. Karlsen, K.A. Lie, and N.H. Risebro. Splitting Methods for Partial Differential Equations with Rough Solutions. European Mathematical Society, Zürich, 2010. MR2662342 (2011j:65002)

[HV03] W. Hundsdorfer and J.G. Verwer. Numerical Solution of Time-Dependent AdvectionDiffusion-Reaction Equations. Springer, Berlin, 2003. MR2002152 (2004g:65001)

[JL00] T. Jahnke and C. Lubich. Error bounds for exponential operator splittings. BIT, 40:735-744, 2000. MR1799313 (2001k:65143)

[Lub08] C. Lubich. On splitting methods for Schrödinger-Poisson and cubic nonlinear Schrödinger equations. Math. Comp., 77:2141-2153, 2008. MR2429878(2009d:65114)

[MSS99] A. Murua and J. M. Sanz-Serna. Order conditions for numerical integrators obtained by composing simpler integrators. Philos. Trans. Royal Soc. London ser. A, 357:10791100, 1999. MR1694703 (2000b:65148)

[Ros63] H. H. Rosenbrock. Some general implicit processes for the numerical solution of differential equations. Comput. J., 5:329-330, 1962/1963. MR0155434(27:5368)

[She89] Q. Sheng. Solving linear partial differential equations by exponential splitting. IMA J. Numer. Anal., 9:199-212, 1989. MR1000457 (90g:65163)

[Suz90] M. Suzuki. Fractal decomposition of exponential operators with applications to many-body theories and Monte Carlo simulations. Phys. Lett. A, 146:319-323, 1990. MR:1059400 (91d:81005)

[Suz91] M. Suzuki. General theory of fractal path integrals with applications to many-body theories and statistical physics. J. Math. Phys., 32:400-407, 1991. MR.1088360 (92k:81096)

[TT95] T. Tang and Z-H. Teng. Error bounds for fractional step methods for conservation laws with source terms. SIAM J. Numer. Anal., 32:110-127, 1995. MR.1313707 (95m:65155)

[Tang98] T. Tang. Convergence analysis for operator-splitting methods applied to conservation laws with stiff source terms. SIAM J. Numer. Anal., 35:1939-1968, 1998. MR.1639974 (99f:65132)

[Tal08] M. Thalhammer. High-order exponential operator splitting methods for timedependent Schrödinger equations. SIAM J. Numer. Anal., 46:2022-2038, 2008. MR2399406 (2009b:65163)

[Yos90] H. Yoshida. Construction of higher order symplectic integrators. Phys. Lett. A, 150:262-268, 1990. MR1078768 (91h:70014) 
Instituto de Matemática Multidisciplinar, Universitat Politècnica de València, 46022 VAlencia, Spain

E-mail address: serblaza@imm.upv.es

Departament de Matemàtiques and imaC, Universitat Jaume I, 12071 Castellón, SPAIN

E-mail address: Fernando.Casas@mat.uji.es

inRia Rennes and Ecole Normale Supérieure de Cachan, Antenne de Bretagne, Avenue Robert Schumann, 35170 Bruz, France

E-mail address: Philippe.Chartier@inria.fr

EHU/UPV, Konputazio Zientziak eta A.A. SAIla, Informatika Fakultatea, 12071 Donostia/San Sebastián, Spain

E-mail address: Ander.Murua@ehu.es 